\title{
GRADUALLY DEVITRIFIED Co-BASED METALLIC GLASS AS A MODEL MATERIAL FOR TESTING NÉEL'S THEORY OF THE RAYLEIGH RULE
}

\author{
H.K. LACHOWICZ ${ }^{a, b}$, F. PoplaWSKI ${ }^{a, b}$, S.N. KANE ${ }^{a *}$, M. KUŹMIŃSKI ${ }^{a}$ \\ AND T. KULIK ${ }^{c}$ \\ ${ }^{a}$ Institute of Physics, Polish Academy of Sciences \\ Al. Lotników 32/46, 02-668 Warszawa, Poland \\ ${ }^{b}$ College of Science, Al. Lotników 32/46, 02-668 Warszawa, Poland \\ ${ }^{c}$ Department of Materials Science \& Engineering, Warsaw University of Technology \\ Narbutta 85, 02-524 Warszawa, Poland
}

(Received November 11, 1998; in final form December 23, 1998)

It is shown in the present paper that gradually devitrified Co-based non-magnetostrictive metallic glass is an excellent model material to verify Louis Néel's theory of the Rayleigh rule. In the course of the calculations, Néel showed that the parameter $p=b H_{c} / a$ (where $H_{c}$ is the coercivity, $a$ and $b$ are the coefficients of a quadratic polynomial expressing the Rayleigh rule) is expected to range between 0.6 (hard magnets) and 1.6 (soft). However, the experimental values of this parameter, reported in the literature for a number of mono- and poly-crystalline magnets, are much greater than those expected from the theory presented by Neel (in some cases even by two orders of magnitude). The measurements, performed for a series of Co-based metallic glass samples annealed at gradually increasing temperature to produce nanocrystalline structures with differentiated density and size of the crystallites, have shown that the calculated values of the parameter $p$ fall within the range expected from Néel theory.

PACS numbers: $75.50 . \mathrm{Kj}, 75.60 . \mathrm{Ej}$

\section{Introduction}

In 1887, Lord Rayleigh [1] discovered an empirical relation which describes the virgin magnetization curve measured in the range of weak applied fields (much lower than the coercivity of a given material), now commonly known as the Rayleigh rule. This relation is given as a quadratic polynomial of the form

$$
M=a H+b H^{2},
$$

where $M$ is the magnetization at the applied field $H$, and $a, b$ are the coefficients characteristic for a given material.

*on a short leave from the School of Physics, D.A. University, Indore, India 
As is commonly known, the first term in the above relation expresses reversible changes of magnetization (the coefficient $a$ is identified with the initial magnetic susceptibility) whereas the second term is irreversible (the coefficient $b$ is known as the Rayleigh constant).

In 1938, Kersten [2] proved Eq. (1). For this purpose he introduced the formalism of the so-called "model of potential function $V(x)$ ". This model assumes that the interaction of a Bloch wall may be described by a conservative potential energy which is a random function $V(x)$ of the coordinate $x$ lying along the direction of the wall motion. Later on, Néel [3], using the same model and assuming that the distribution of the pinning forces follows a Gaussian probability law, improved the calculations made by Kersten. Under this assumption, Néel, combining the calculated values of the coefficients $a, b$ and of the coercivity, $H_{\mathrm{c}}$, derived a dimensionless parameter $p=b H_{c} / a$, the value of which is expected to range between 0.6 (hard magnetic materials) and 1.6 (soft materials) [4]. Comparison of the experimental and calculated values of the parameter $p$ is usually considered as a practical numerical test for the validity of the model used by Néel. However, the experimental values of this parameter, measured till now for numerous monoand poly-crystalline soft and hard magnets, are much larger than those expected from Néel's theory (see, e.g., [4-6]). For example, in the experiment performed by Porteseil [4] on $\mathrm{Fe}-\mathrm{Si}$ single crystal rectangular frames, each containing a single active Bloch wall, the value of the parameter $p$ was obtained as 260 which is more than two orders of magnitude larger than the expected value of 1.6.

As it has been claimed by Porteseil [4], this giant divergence results mainly from long-range correlations in the random energy landscape existing in real magnets in which domain walls move, contrary to Néel's theory in which statistical correlations in $V(x)$ are neglected. Because of these correlations, the probability of a wall jumping across several energy barriers at the time of a single jump may dramatically increase, thus enhancing the irreversible contribution to the magnetization, and consequently the coefficient $b$.

To the best of our knowledge, the only value of the parameter $p$ (equals 1.4) which was in agreement with the theoretical expectation, was obtained in the experiments of Porteseil and Geoffroy on rapidly quenched Co-based amorphous ribbon specimens [7]. They have taken into account the fact revealed by transmission electron microscopy [8] that $\mathrm{Fe}$ - and Co-based amorphous ribbons can contain nanocrystals of $\alpha-\mathrm{Fe}$ (a typical size is around $10 \mathrm{~nm}$ ) even in their as-quenched state. Considering that the anisotropy of amorphous magnets is extremely small and that the magnetostriction of the Co-based alloys used in the experiment vanishes, it has been assumed [7] that the major contribution to the wall pinning is the anisotropy energy of the nanocrystallites inside the wall.

Taking into account the experiment described above, it may be expected that the parameter $p$, calculated from the data obtained for a series of Co-based amorphous ribbon specimens gradually devitrified in a controlled way to produce intentionally nanocrystalline structure with differentiated density of these nanograins, should verify Néel's theory in a more satisfactory way. iment.

The aim of the work presented in this paper was to carry out such an exper- 


\section{Experimental}

For the experiment, a rapidly quenched amorphous ribbon of nominal composition $\mathrm{Co}_{71} \mathrm{Fe}_{1} \mathrm{Mo}_{1} \mathrm{Mn}_{4} \mathrm{Si}_{14} \mathrm{~B}_{9}$ (Vitrovac 6030 produced by Vacuumschmelze, $\mathrm{GmbH}$ ) was used as the parent material. Extremely low magnetostriction, which this material exhibits $\left(<0.2 \times 10^{-6}\right)$, was the main reason for the choice. This feature of the material is crucial in the case considered since for samples showing non-negligible magnetostriction, the coercivity will be mainly determined by magnetoelastic energy created by long-range correlated stress field. Therefore, a reliable determination of the influence of the nanocrystallites generated would not have been possible.

The specimens used were $25 \mu \mathrm{m}$ thick, $5 \mathrm{~mm}$ wide and $80 \mathrm{~mm}$ long. All the samples were annealed for 1 hour at temperatures ranging from 420 up to $600^{\circ} \mathrm{C}$. To prevent eventual surface oxidation, all the samples were kept in evacuated quartz ampoules during annealing (as shown in Ref. [9], annealing in air even at temperatures as low as $250^{\circ} \mathrm{C}$ leads to the formation of surface oxides, non-magnetic borosilicate $\mathrm{B}_{2} \mathrm{O}_{3}+\mathrm{SiO}_{2}$ ).

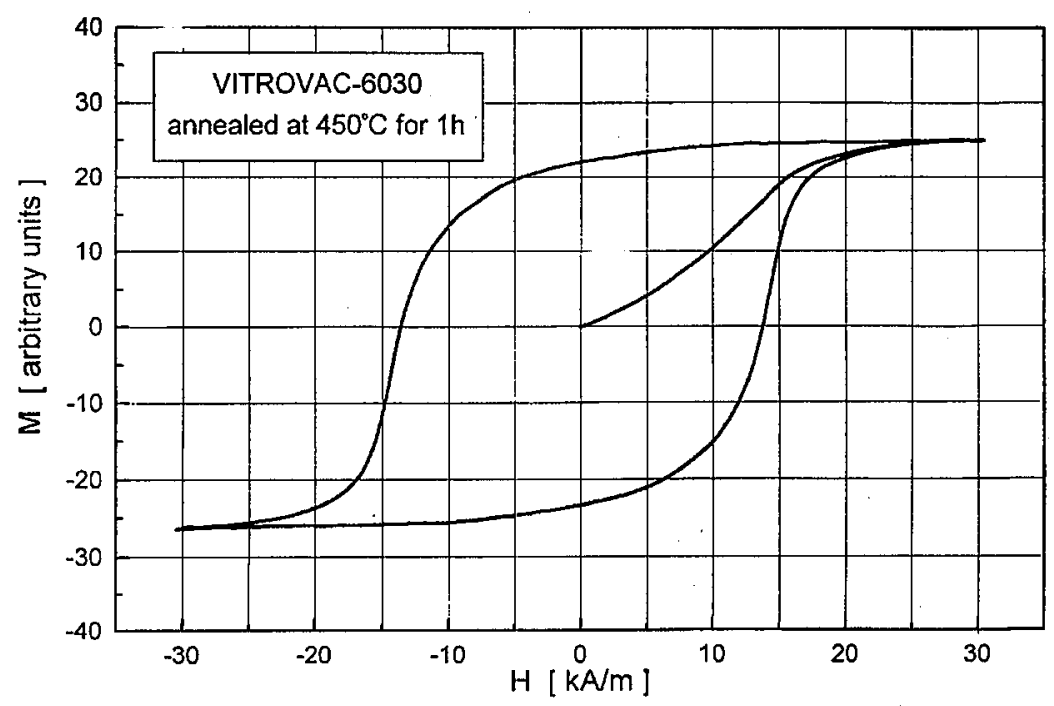

Fig. 1. A typiçal hysteresis loop is shown along with the virgin magnetization curve.

The virgin magnetization curves as well as the hysteresis loops at saturation have been measured along the sample axis by means of a conventional inductive technique in a computerized high sensitivity set-up operating at a frequency $25 \mathrm{~Hz}$ of the sawtooth magnetizing field. Before measurement each sample was demagnetized by an a.c.-field. A typical magnetization characteristic obtained in the experiment is shown in Fig. 1. The facility mentioned above also makes it possible to measure magnetization characteristics at elevated temperatures up to about $400^{\circ} \mathrm{C}$ by using an electric furnace kept coaxially inside the magnetizing solenoid.

The microstructure of the annealed specimens was studied by means of transmission electron microscopy (Philips EM-300, operating at $100 \mathrm{keV}$ ). 
Magnetic domain structures have also been observed in the samples by means of the Kerr-effect technique using a computerized set-up which makes it possible to cancel any non-magnetic background. The samples were always demagnetized by an a.c.-field before observing the domain structures.

\section{Results and discussion}

Figure 2 shows the dependence of the coercivity of the samples as a function of the annealing temperature. In the same figure the dependence of the parameter $p=b H_{\mathrm{c}} / a$ on the temperature is also presented. The values of this parameter have been computed using the values of the coercivity determined from the hysteresis loops and the values of the coefficients $a$ and $b$ obtained from the best fit (least squares method) to Eq. (1) of the virgin magnetization curve. The error in calculations of the values of the parameter $p$, resulting from the calculated values of the coercivity from the measured hysteresis loops and from those obtained on the ground of the fitting procedure for the parameters $a$. and $b$, is estimated to be not greater than $20 \%$. As seen in Fig. 2, the calculated values of the parameter $p$ fall within the range predicted by Néel's theory $(0.6<p<1.6)$. This result shows that owing to the invention of metallic glasses and later on, nanocrystalline magnets for which metallic glasses serve as the parent materials, it is possible to produce magnets which behave in a way anticipated by Néel in his theory of the Rayleigh relation.

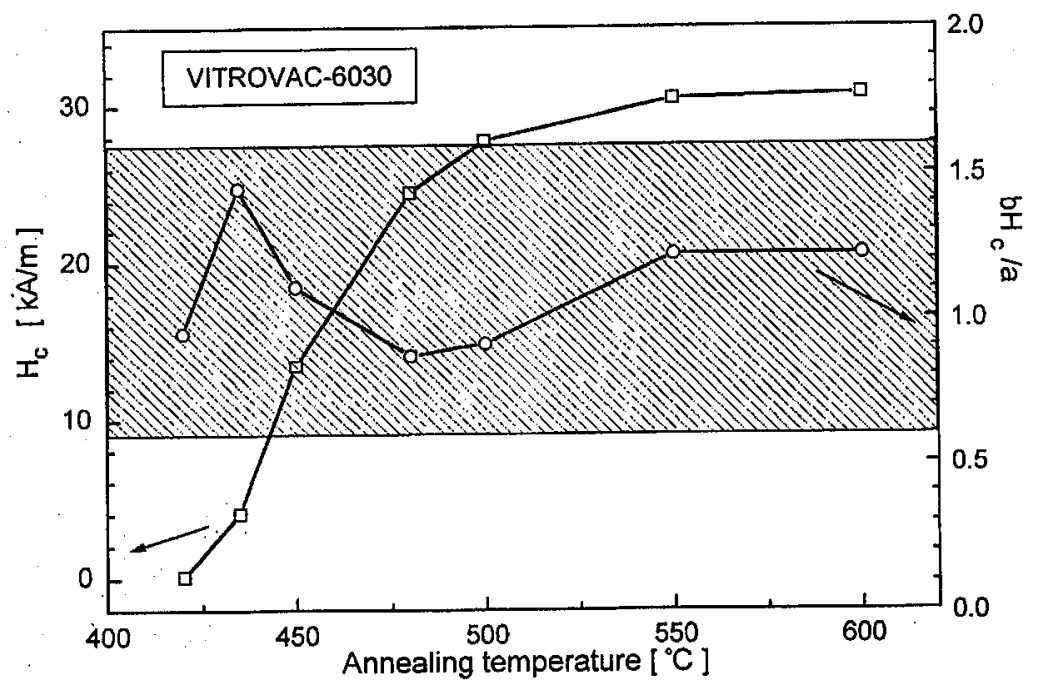

Fig. 2. Annealing temperature dependences of the coercivity and the parameter $p$ (hatched area shows a theoretical limitation of the parameter $p$ ).

Figure 2 shows that the parameter $p$ varies with the annealing temperature in a rather erratic fashion. According to Néel's theory, this parameter should evolve monotonically, smoothly decreasing with the increase in coercivity. At higher annealing temperatures the volume, density of the crystallites formed can be high 
enough so that the assumption on non-correlated random energy landscape becomes invalid. An increase in the value of $p$, observed at higher annealing temperatures (see Fig. 2), seems to verify the above explanation since an increase in the value of $b$ is then expected.

It should, however, be noticed that in the range of low fields, irregular deviations from the parabolic shape have been observed (similar irregularities have been reported by Koller et al. [10] in a similar experiment performed on a polycrystalline nickel specimen). On the other hand it has been found that for the samples investigated, the Rayleigh rule holds in a relatively wide range of the external magnetizing fields, approximately up to the half of the value of the coercivity for a given sample.

In order to test the accuracy of the fit to Eq. (1), the measured virgin magnetization curves have also been approximated by a cubic polynomial by adding a third term $c H^{3}$ to Eq. (1) (it has been shown by Porteseil [4] that such a polynomial well describes the virgin curve measured for $\mathrm{Fe}-\mathrm{Si}$ single-crystal sample). The fit has shown that the coefficient $c$ is at least three orders of magnitude smaller than the coefficients $a$ and $b$. Since the cubic term reflects the deviation from the Rayleigh rule, the above relation between the coefficients $a, b$ and $c$ shows that the measured virgin magnetization curves can be described with a sufficient accuracy by a quadratic polynomial, indicating that the Rayleigh rule is indeed satisfied. Figure 3 presents a typical measured virgin magnetization curve and its best fit to Eq. (1).

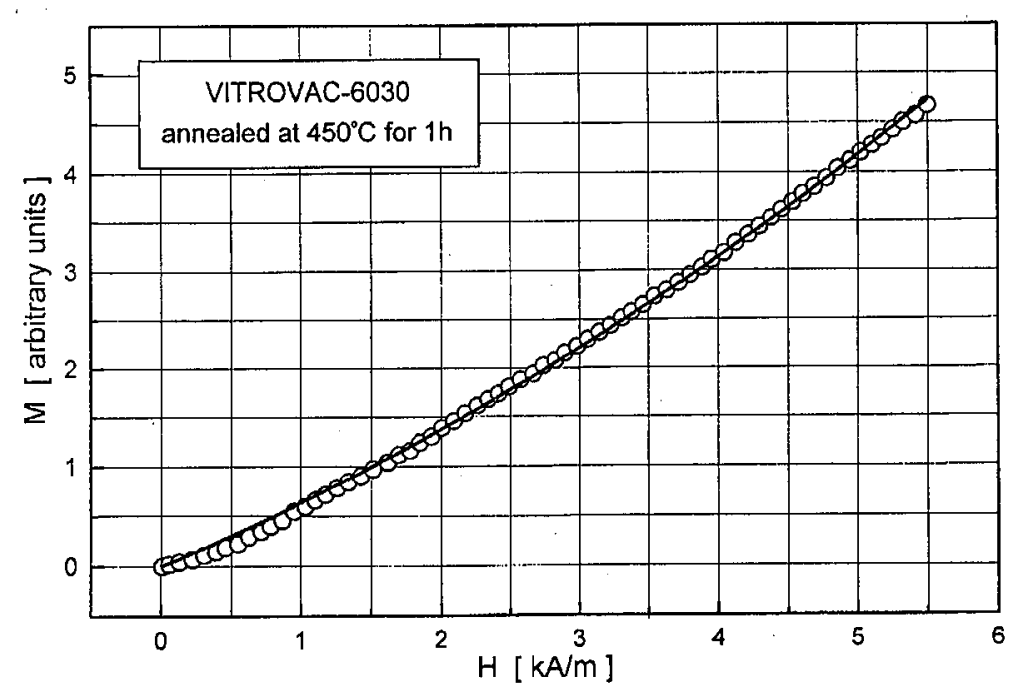

Fig. 3. A typical virgin magnetization curve; the solid line gives the best fit of the experimental points to the Rayleigh rule (quadratic polynomial).

As seen in Fig. 2, an increase in the annealing temperature causes a rapid rise of the coercivity. In the range of these temperatures from 420 up to $600^{\circ} \mathrm{C}$, the coercivity increases by more than two orders of magnitude. This effect is however 

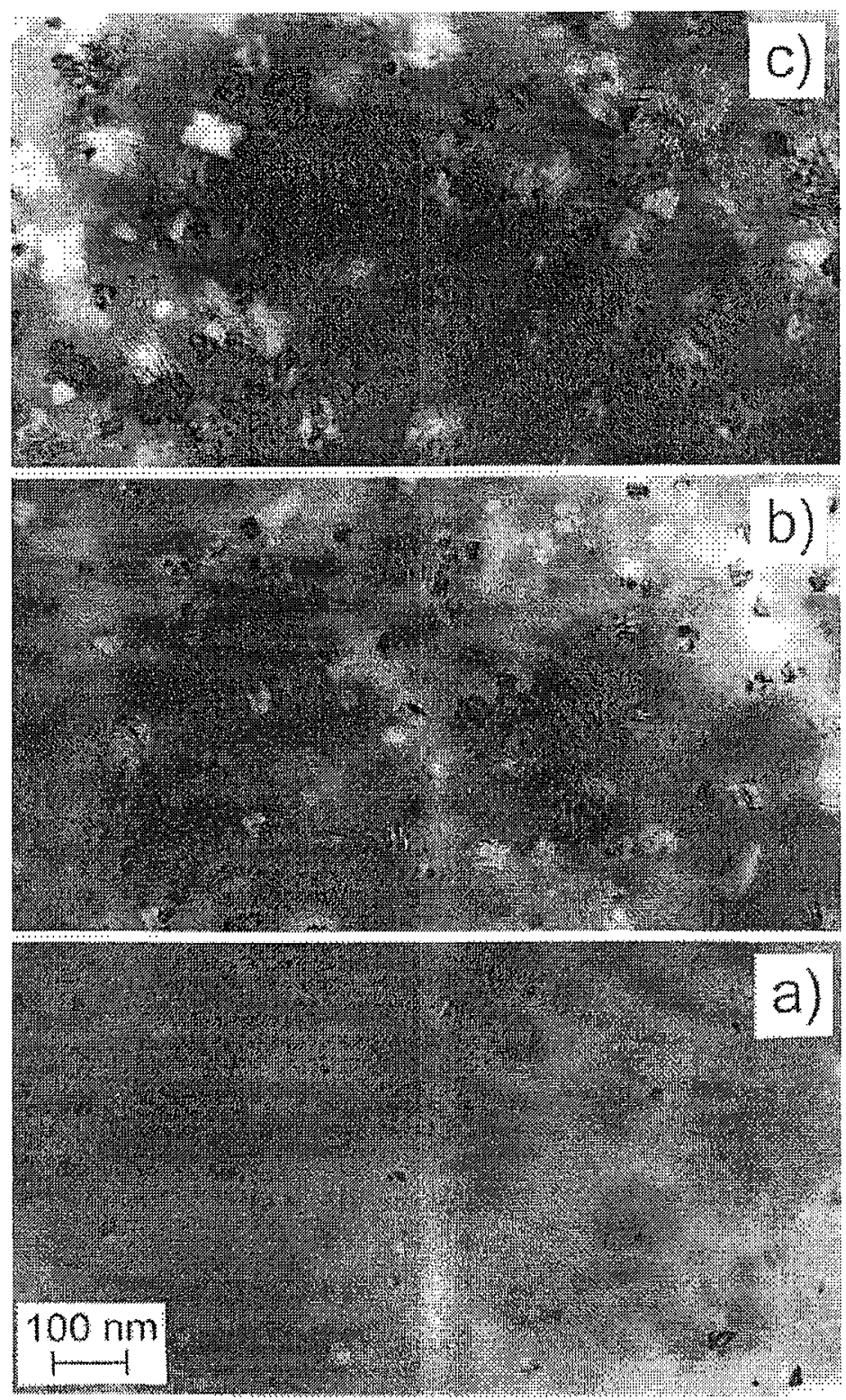

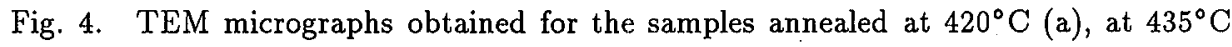
(b) and at $480^{\circ} \mathrm{C}$ (c). The magnification is the same for all the micrographs.

quite obvious since higher the temperature of annealing, the larger is the density of the crystallites formed, leading to an increase of the pinning forces which retard the wall motion. As seen in Fig. 4, which shows TEM-micrographs obtained for the samples annealed at various temperatures in the range where a rapid increase in the coercivity is observed, the density and the mean size of the crystallites increase with the temperature of annealing, as expected. 
An expression for the coercivity in a non-magnetostrictive, amorphous medium containing randomly distributed, non-interacting nanocrystallites has been derived by Porteseil and Geoffroy [7] and is given in the following form:

$$
H_{\mathrm{c}}=n^{1 / 2} \delta^{-3 / 2} K \nu / 12 M_{\mathrm{s}}
$$

where $n$ is the volume density of nanocrystallites, $\delta$ - the wall width, $K-$ the anisotropy constant of nanocrystallites, $\nu$ - the mean volume, and $M_{\mathrm{s}}-$ the magnetization of the sample.

It can be easily noticed from the above expression that the coercivity should rise with an increase in density and size of the crystallites, assuming that the wall width is mainly determined by the properties of the amorphous matrix and, therefore, does not change significantly.

However, it should be noticed that annealing the samples at higher and higher temperatures leads to an increase in the volume density of the crystallites as well as to an increase in their mean size. As a consequence, the composition of the matrix undergoes some changes. Similarly, the composition of the crystallites can vary since they are most probably composed of Co-based solid solution (see Ref. [11]). Both these lead to uncontrolled changes of the magnetic properties of both phases. Since Néel's theory has been formulated for a material of well-defined properties, it is not surprising that the dependence of the parameter $p$, obtained for a series of samples gradually devitrified, does not follow precisely the behavior one expects theoretically.

The value of the parameter $p$ as well as its changes can be observed for a nanocrystalline sample for which the virgin magnetization curve and its coercivity are measured as a function of temperature. Such a procedure should allow one to avoid eventual changes of the composition of both phases, amorphous and crystalline, assuming that the temperature of measurement is lower than the temperature of crystallization. It can be expected that the results obtained this way would be more reliable than those from a series of samples annealed at different temperatures. Figure 5 presents the measuring temperature dependences of the coercivity and the parameter $p$ for the sample annealed at $450^{\circ} \mathrm{C}$. As seen in this figure, the coercivity monotonically decreases with an increases in temperature of measurement from 30 up to $200^{\circ} \mathrm{C}$, whereas, the parameter $p$ increases in this temperature range. The coercivity scales with the quotient of anisotropy and magnetization. The anisotropy usually decreases with temperature much faster than magnetization, in particular, in the temperature range much lower than the Curie point and this fact can be taken into account to interpret the observed drop of coercivity. Figure 5 also shows that the value of the parameter $p$ increases with a decrease in the coercivity. Such a dependence is, in principle, in agreement with Néel's theory which anticipates an increase in $p$ with a decrease in coercivity. However, the two-fold variation of this parameter, while the change of coercivity is only $25 \%$, is not consistent with the theory. The values of $p$ give, however, the right order of magnitude (see Fig. 5):

Néel's model describes in principle the motion of a single Bloch wall. The experiments, which have been used to calculate the parameter $p$, were usually carried out using samples with a rather complex domain structure (except those 


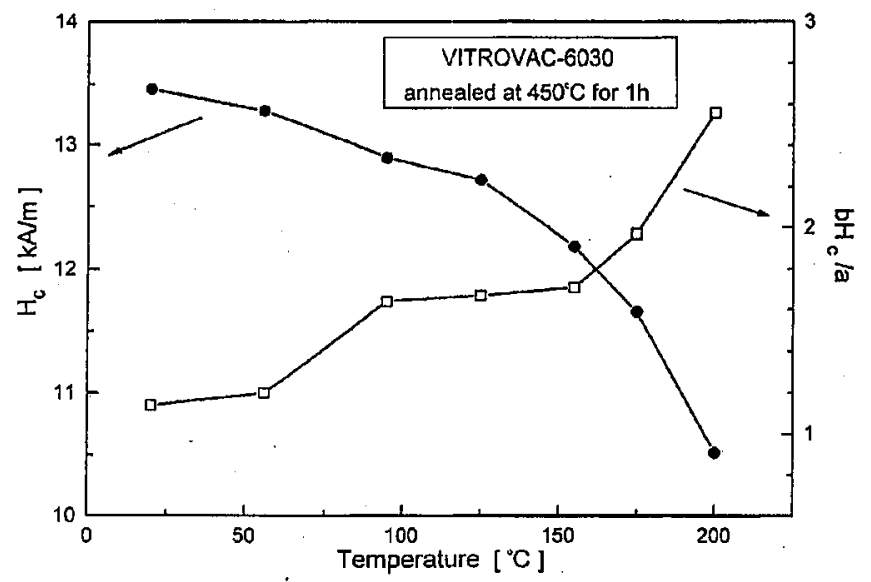

Fig. 5. Dependences of the coercivity and the parameter $p$ as a function of the temperature of measurement.
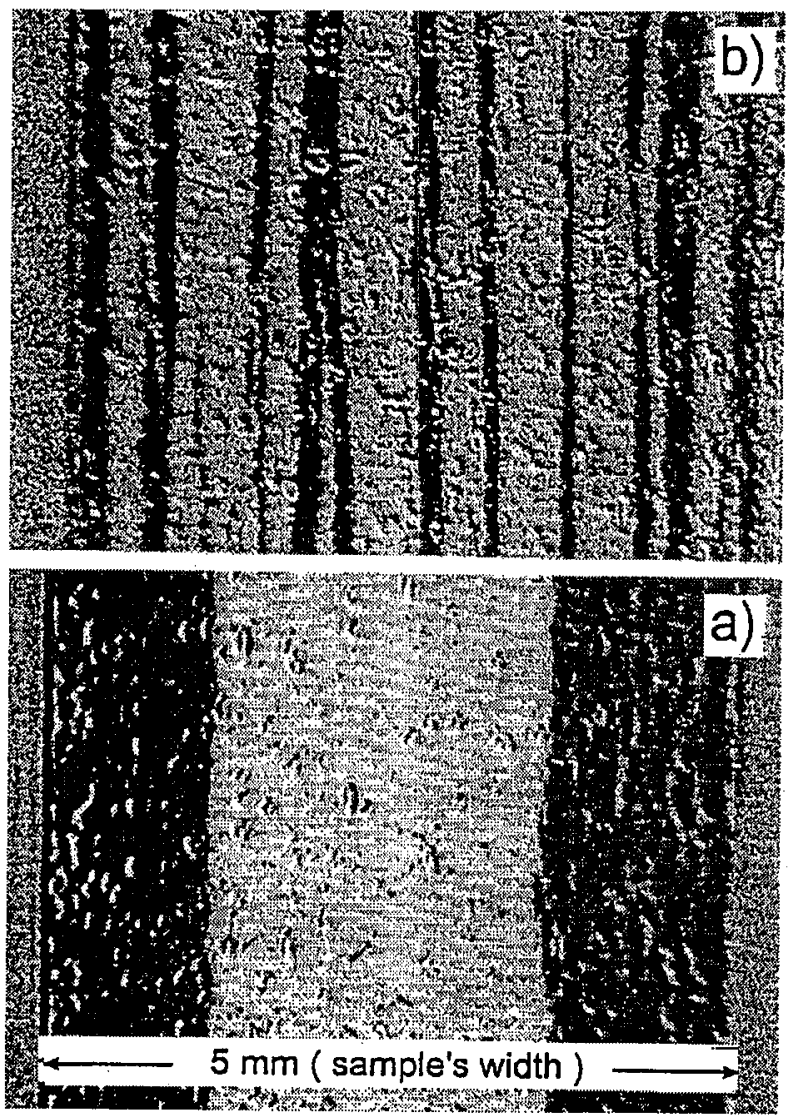

Fig. 6. Domain structures for the relaxed sample annealed at $400^{\circ} \mathrm{C}$ (a) and for the sample annealed at $420^{\circ} \mathrm{C}$ in which nanocrystallites just appear (b). 
performed on single-crystal framed samples). This fact may also be the reason for the large disagreement between the experimental and the theoretically expected values of this parameter. In the case of the samples used in the present work, the domain structure in the demagnetized state is very simple and consists of domain walls directed along the ribbon length. The number of these domains increases with an increase in the volume density of the crystallites; the effect is not surprising since the pinning force becomes stronger for a larger density. Such a configuration of domains results in a negligible rotational magnetization processes which do not disturb the results of measurements in a significant way. The change of the domain pattern which follows the sample after annealing at high enough temperatures, to create a perceptible nanocrystalline structure, is clearly visible in Fig. 6 . Here the pattern "for the relaxed sample (annealed at $400^{\circ} \mathrm{C}$ ) is shown (Fig. 6a) and also that for the sample annealed at $420^{\circ} \mathrm{C}$ (Fig. 6b), the temperature at which the nanocrystalline structure just appears (see Fig. 4a which presents the micrograph of the crystallographic structure of this sample).

The experimental results shown above allow one to consider this series of nanocrystalline magnets as a suitable model material to verify Néel's theory of the Rayleigh rule. The basic property of this model material is that the only obstacle for the moving walls are the nanocrystallites which influence this movement through their anisotropy energies and that these energies can be treated as statistically independent random variables. It is not surprising since the nanocrystallites formed grow randomly so that their crystallographic directions are also randomly distributed. Owing to the exchange coupling at the crystal-matrix interfaces, the magnetization of the crystallites is forced to be aligned along that inside the surrounding matrix. If there is no direct magnetic interaction between the crystallites, the orientations of the easy axes of magnetization are independent and consequently the anisotropy energies are independent variables thus fulfilling the assumptions made by Neel in his theory.

\section{Conclusions}

It has been shown that gradually devitrified Co-based metallic glass is an excellent model material to verify the validity of Neel's theory describing the Rayleigh rule. The results presented show for the first time that the parameter $p$, usually considered as a numerical test for the validity of the model used by Néel, exhibits values which lie within the theoretically expected range $0.6<p<1.6$.

\section{Acknowledgments}

The work was supported in part by the Committee for Scientific Research (Poland) under grant No. 8 T11B 048 10. The authors are greatly indebted to Prof. J.L. Porteseil (Louis Néel Laboratory, CNRC, Grenoble, France) for helpful discussions and for supplying the reprints of Néel's early papers. Thanks are also due to Dr. G. Herzer (Vacuumschmelze, GmbH, Hanau, Germany) for supplying the Vitrovac 6030 amorphous ribbon. 


\section{References}

[1] Lord Rayleigh, Philos. Mag. 23, 225 (1887).

[2] M. Kersten, Phys. Z. 9, 860 (1938).

[3] L. Néel, Cah. Phys. 12, 1 (1942); 13, 18 (1943).

[4] J.L. Porteseil, Phys. Status Solidi A 51, 107 (1979).

[5] B. Astie, J. Degauque, J.L. Porteseil, R. Vergne, J. Magn. Magn. Mater. 28, 149 (1982).

[6] A.R. Ali, G. Said, Physica B 112, 241 (1982).

[7] J.L. Porteseil, O. Geoffroy, J. Magn. Magn. Mater. 140-144, 1855 (1995).

[8] E. Ferrara, F. Fiorillo, M. Pasquale, A. Stantero, M. Barrico, C. Béraund, J. Degauque, B. Viala, E. de Trémolet du Lacheisserie, J.L. Porteseil, Key Eng. Mater. 81-83, 189 (1993).

[9] C.K. Kim, R.C. O'Handley, Mater. Sci. Eng. B 38, 16 (1996).

[10] A. Koller, E. Pfrenger, K. Stierstadt, J. Appl. Phys. 39, 869 (1968).

[11] H.K. Lachowicz, T. Kulik, R. Żuberek, L. Małkiński, M. Kuźmiński, A. Ślawska-Waniewska, J.S. Muñoz, J. Magn. Magn. Mater. 190, 267 (1998). 